\title{
Novel Cocrystallization of Apolipoprotein A-I with Butyric Acid Grace Ferri ${ }^{1}$ \\ ${ }^{1}$ No affiliation given \\ grace.ferri@comcast.net
}

Diseases of lipid metabolism, in particular cardiovascular atherosclerosis, remain the number one cause of morbidity and mortality in Western society. The well-documented anti-atherogenic role of high-density lipoprotein (HDL) is related to its involvement in reverse cholesterol transport (Fielding 211). When the transporter ABCA1 initiates cholesterol efflux from peripheral and hepatic cells, apolipoprotein A-I (apoA-I) binds the free cholesterol together with phospholipid to form discoidal nascent HDL ("good cholesterol"). The enzyme lecithin:cholesterol acyltransferase (LCAT) catalyzes the esterification of the cholesterol carried by HDL. ApoA-I activates LCAT for reverse cholesterol transport, wherein LCAT esterifies the cholesterol within the nascent HDL2 and facilitates maturation of HDL to a mature spherical form. After esterification, cholesterol can no longer diffuse back to the peripheral and hepatic cells. The esterified cholesterol, or cholesteryl ester, molecules form the hydrophobic core of the mature HDL particle and leave the circulation upon uptake by a hepatic scavenger receptor for excretion by the liver.

Research in Dr. Atkinson's laboratory implicated the arginine 123 residue of apoA-I in the function of LCAT (Gorshokova 348). Based on the X-ray crystal structure, the Arg123 residues of each apoA-I monomer enclose a hydrophobic tunnel into which the lipid substrates of LCAT may be sequestered. ApoA-I has a closed conformation without lipid bound that transforms to a "double belt" encircling the nascent HDL disc (Segrest 31755; Mei 38570). When apoA-I activates LCAT, the tunnel should widen in order to present the substrates to LCAT for esterification (Gorshokova 348). However, the mechanism by which apoA-I interfaces with LCAT substrates remains unknown. Cocrystallization of apoA-I with the lipid component, butyric acid, will enable us to assess how the "double belt" of apoA-I opens in three-dimensional space. Ultimately, research into the activation of LCAT by apoA-I should shed light on the atheroprotective mechanism of HDL and the prevention of cardiovascular disease (Xia 33143; Rosenson 1905; Gorshokova 348). 